Original Research Paper

\title{
A Firefly-Inspired Scheme for Energy-Efficient Transmission Scheduling Using a Self-Organizing Method in a Wireless Sensor Networks
}

\author{
Zeyad Ghaleb Al-Mekhlafi, Zurina Mohd Hanapi, \\ Mohamed Othman and Zuriati Ahmad Zukarnain \\ Department of Communication Technology and Network, Faculty of Computer Science and Information Technology, \\ Universiti Ptura Malaysia, UPM 43300, Serdang, Malaysia
}

Article history

Received: 27-09-2016

Revised: $30-11-2016$

Accepted: 10-12-2016

Corresponding Author: Zeyad Ghaleb Al-Mekhlafi

Department of Communication Technology and Network,

Faculty of Computer Science and Information Technology, Universiti Ptura Malaysia, UPM 43300, Serdang, Malaysia

Email: ziadgh2003@hotmail.com

\section{Introduction}

The emerging research trend in the areas of MicroElectrical Mechanical Systems (MEMS) over the years has provoked scholarly attention on Wireless Sensor Networks (WSNs). These networks are small, inexpensive devices that are capable of processing, sensing and transmitting the ecological phenomenon of attention. WSNs have different function forecast in armed, manufacturing and undeveloped observing systems (Al-Mekhlafi et al., 2014b). In SNs, the wireless data sensors cooperatively intelligence the surroundings, become aware of the occurrence of attention and finally ahead the data to an enthusiastic BS in time synchronization. The time synchronization among the sensor nodes is necessary for the harmonization of authority cycles, which ensures energy-efficiency and for the steady performance of data sensors in real time observing scenarios. One of the methods which might be working to scheme WSN performance is the PCO scheme, which has been utilized to model the flashing-light-emittingattracting mating partners. However, WSNs are not capable to send and obtain sensor data simultaneously and for mainly situations, battery substitute is not possible in the lead exhausting the battery energy of a sensor node. Therefore, energy-efficiency procedures (Al-Mekhlafi et al., 2014a; Wang et al., 2012) are vital design requirements for WSNs. an additional significant necessity of WSNs is their self-organization ability that allows SNs to find again their novel neighbors (because of battery tiredness or rapid break down of some sensor nodes in the network) amid self-motivated network topology alters. Energy-efficient is the main concern in designing SN because of the limited and nonrechargeable power resource. The research areas on the energy efficiency of SNs are classified into routing, localization, time synchronization, clustering, data 
aggregation and security (Fig. 1). Therefore, this study focuses on the energy consumption at the transmission scheduling for time synchronization on WSNs during the transmitting (sender) state.

We suggest a firefly-inspired self-organizing method to ensure a highly energy-efficient control mechanism in WSNs in the case of connection failures (i.e., deafness or collision caused by the movement of SNs or the depletion of SN energy). By determining the reliability of the captured data to be reported to the sink node, the proposed mechanism reduces the transmission delay and consumed energy of the SNs. The major goal of the proposed scheme is to expand a self-organizing time synchronization algorithm by adopting the PCO model following the synchronous flashing of fireflies to counteract deafness and collision (Al-Mekhlafi et al., 2014b) in addition to decrease the energy usage of the SNs.

The remainder of this paper is organized as follows. Section 2 appraisals some of the related work, section 3 depicts the scheme patterns that are utilized in the proposed method, section 4 briefly discusses the purpose and major processes of the proposed method, section 5 shows the performance assessment of the proposed method and section 6 summarizes the paper.

\section{Literature Review}

This section reviews the background of the energy efficiency of transmission scheduling considering deafness and collision avoidance by using a selforganizing method in WSNs. This transmission scheduling is commonly applied for WSNs. Energy- efficient is guaranteed in SNs by explicitly embedding power minimization protocols interested in the fundamental intelligence scheme of the sensors data (for instance plummeting the per packet power usage) (Nokhanji et al., 2015) or bypassing the elevated power consumption of some solitary sensor node inside the network. Therefore, the broadcast scheduling problems in clock synchronization on WSNs are divided into sender and receiver (Fig. 1). The transmission scheduling problems at the sender include collision, deafness and hidden terminal. Packet collision usually occurs when a SN misses the control packet, concludes that the channel is idle and then attempts to transmit on the channel while another node is busy transmitting on the same channel, resulting in the collision and dropping of two packets. Thus, none of the packets are successfully received. Deafness refers to the phenomenon in which the nodes are unable to receive data during transmission. Hidden terminal refers to the situation in which a sensor node efforts to broadcast a packet to an additional sensor node that is already sending packets to another node that is unknown to the attempting node, thereby leading to the collision of the two packets. The hidden node problem is solved in multichannel networks because the receiving node can tune its receiver to another channel different from the channel of its transmitter. The broadcast scheduling problems at the recipient include idle listening and overhearing. Idle listening refers to the situation in which a sleeping node wakes up only to find no active transmissions, while overhearing refers to the reception of data packets by a node that is not the intended recipient.

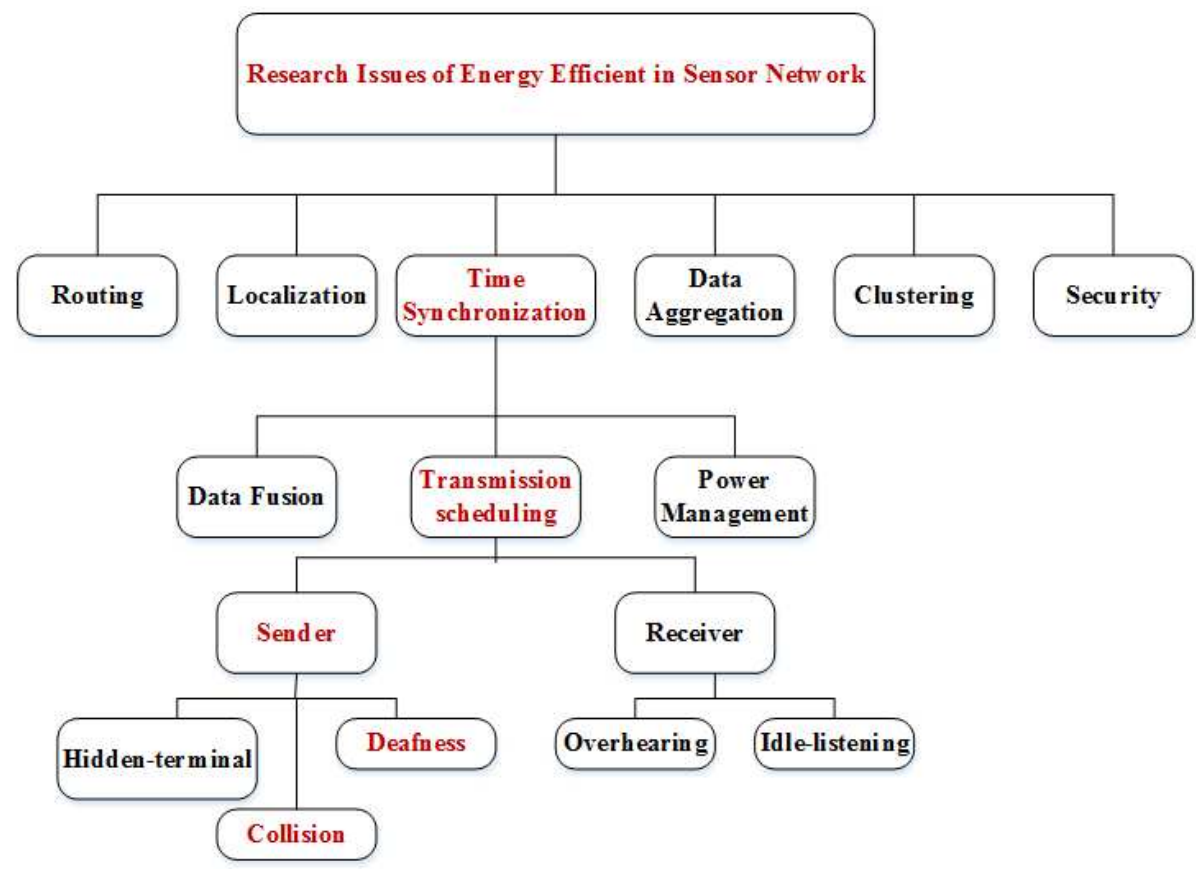

Fig. 1. Categorization of research issues on the energy-efficient in sensor networks 


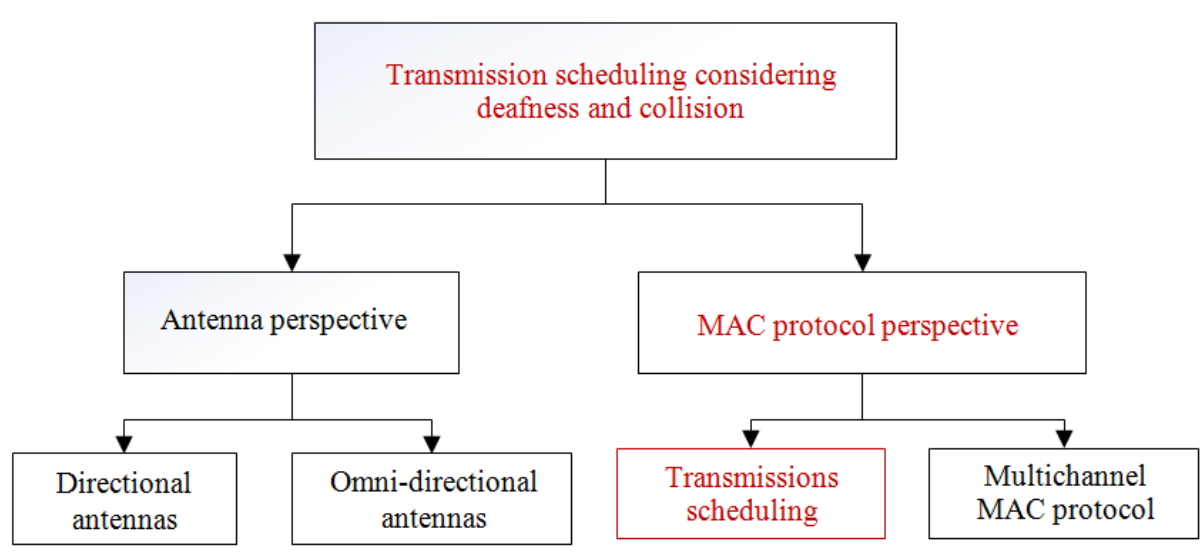

Fig. 2. Transmission scheduling in WSNs

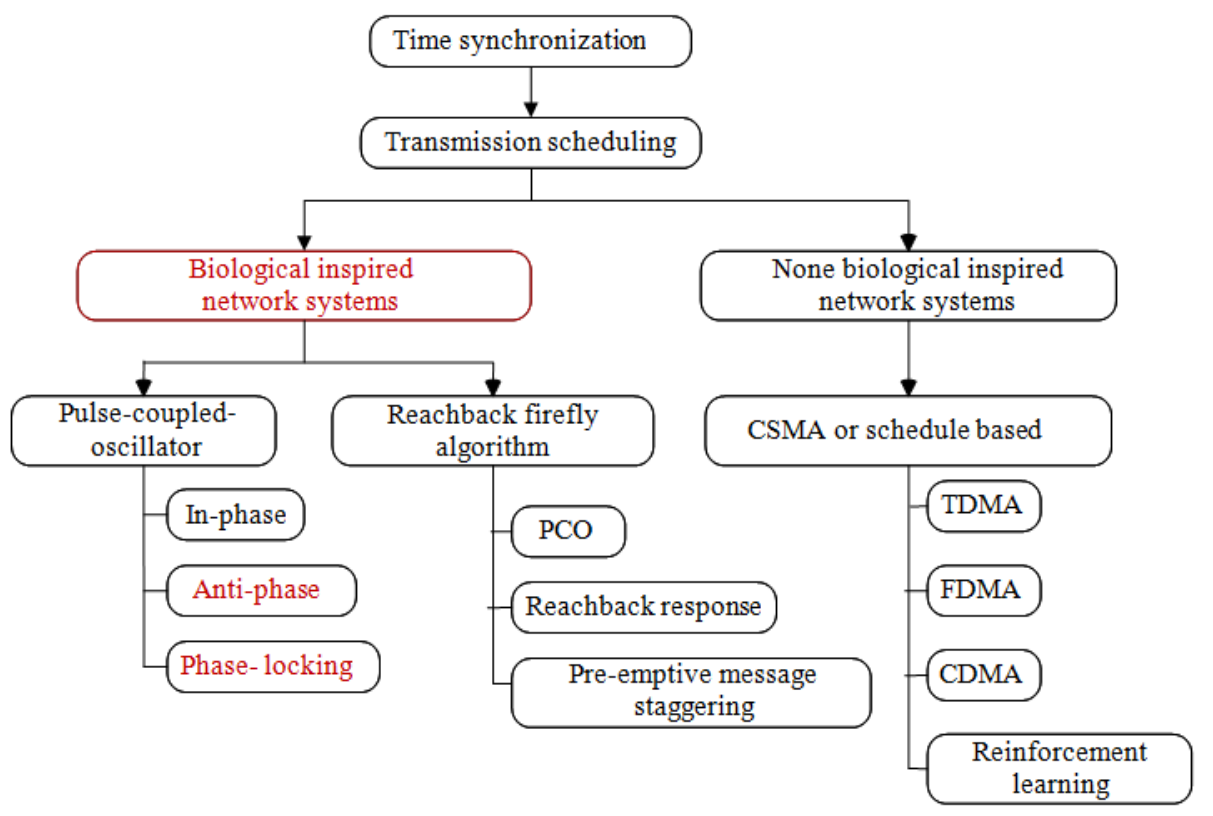

Fig. 3. Classification of the Transmission Scheduling Mechanisms in WSN

The above mentioned problems require the coordination of transmission scheduling in time synchronization for WSN among SNs (Al-Mekhlafi et al., 2014a; 2014b) provide a detailed review and classified the transmission scheduling that considers deafness and collision avoidance in time synchronization for WSN.

Figure 2 presents more details on the transmission scheduling that considers deafness and collision avoidance in time synchronization for WSN.

As shown above, transmission scheduling considering deafness and collation in WSNs can be divided into two perspectives. The first perspective is the antenna perspective, which is further classified into directional and omnidirectional antennas. The second perspective is the MAC protocol perspective, which is further divided into transmission scheduling (MAC protocol) and multichannel MAC protocol perspective. This paper will focus on transmission scheduling (MAC protocol) for the MAC protocol perspective. Several mechanisms for transmission scheduling considering deafness and collision have been proposed by using time synchronization on WSN. These mechanisms are classified into two categories, namely, non-biologically inspired network systems and biologically inspired network systems. Figure 3 shows the proposed mechanisms for transmission scheduling for WSN. This paper will focus on biologically inspired network systems (Nunez et al., 2012) that are based phaselocking and anti-phase in the PCO scheme.

PCO scheme is used to give details the synchronous performances of the in nature inspired network systems that are classified into three neurons as observed in the 
flashing synchronization behaviors of fireflies and pacemaker cells. This paper shall concentrate on the PCO model that is based the synchronous alternating of the synchronization performances of fireflies. Fireflies are beetles, the females of which are unable to fly. A large number of fireflies collect on plants at sunrise and establish emitting light to attract mating partners. The behavior of fireflies is able to be imitated for the growth of WSNs. Similar to SNs; fireflies have decentralized performance, have incomplete individual dispensation ability and are mostly restricted in their announcement. Therefore, biologically inspired firefly models can be categorized either as biological models or mathematical models (Buck, 1988; Mirollo and Strogatz, 1990). These schemes have been working in different WSN mechanism for inter-node announcement (Tyrrell et al., 2007; Werner-Allen et al., 2005). Additional works have employed these schemes to provide time triggered services (Leidenfrost and Elmenreich, 2008) and have been argued lengthily in numerous researches. For instance, Buck et al. (1981) investigated the behavioral prototype of fireflies with admiration to their emission of outside light pulses. Mirollo and Strogatz (1990) residential a mathematical scheme for mimicking firefly time synchronization by using PCO. Their sheme was mostly based two significant processes:

- Each entity independently maintains a periodic schedule of flashing instances

- The model is fine tuned by aligning this model with the coupling parameters and the prevalent magnitude of the internal function to achieve external flashing. Following these steps, network synchronization is achieved as long as certain constraints on the coupling are addressed (Mirollo and Strogatz, 1990)

Several variants of the model of Mirollo and Strogatz have been proposed by different wireless communication researchers. These variants include works on channel mitigation and noise (Hong and Scaglione, 2005), distribution delays (Mathar and Mattfeldt, 1996), effect of network topology on coupling function and network convergence (Lucarelli and Wang, 2004) and pulsebased network synchronization (Tyrrell et al., 2006; Werner-Allen et al., 2005). Nevertheless, since all of these studies distinguish the gaining, network time synchronization and announcement phases in which the data broadcast and reception occur, the proposed variants are inappropriate for WSNs due to these networks are flat to rapid topology alters.

\section{The Scheme Models for PCO}

As mentioned earlier, WSNs are associated with strict energy and delay requirements. Therefore, the proposed method has to provide for these dynamics to achieve a victorious completion as well as to avoid deafness and collision problems, which will subsequently enhance the packet drop rate and network lifetime. This section describes the PCO technique and adopted a PCO model for WSNs that are utilized in this study.

\section{Original PCO Model}

The PCO technique engrosses the time synchronization performance of a number of oscillators, in which an oscillator fires only when its timer arrives at 1 . The customary PCO scheme explains a number of synchronous firefly behaviors, explicitly, in-phase, anti-phase and phase locking, according to PCO synchronization. The in-phasebased scenario completely synchronizes the oscillator, the anti-phase-based scenario ensures the synchronization of the oscillator at equal intervals and the phase-locking-based PCO synchronization (Nishimura, 2015) explains the time synchronization of the oscillator with a stable offset. The traditional PCO model is illustrated in (Goel and Ermentrout, 2002; Mirollo and Strogatz, 1990). Figure 4 presents a flowchart of the original PCO model that explains the algorithm.

By a total $\rho\left(\varnothing_{j}\right)$ as pursues:

$$
\Phi_{j}=\Delta\left(\Phi_{j}\right)+\Phi_{j}
$$

where, $\Delta\left(\Phi_{j}\right)$ is term of the Phase Response Curve (PRC) (Canavier and Achuthan, 2010). PRC is extensively used by experimentalists to enumerate the performance of the system devoid of knowing the fundamental schemes that are accountable for the performance.

The proof of Equation 1 is presented as follows:

- $\quad$ Quadratic-Integrate and Fire (QIF) model (Brunel and Latham, 2003):

$$
\Delta_{\text {OIF }}(\Phi)=a(1-\cos 2 \Pi \Phi)
$$

where, $a=0.5,1.0,-0.5$ which shows in Fig. 5 .

- Radial-Isochron-Clock (RIC) model (Goel and Ermentrout 2002):

$$
\Delta_{R I C}(\Phi)=-a * \sin 2 \Pi \Phi
$$

where, $a=a=0.5,1.0,-0.5$ which shows in Fig. 6 . In this model, an oscillator ignores all stimuli at the total of firing and an oscillator identify multiple stimuli that are received simultaneously as one stimulus.

- The following PRC-Satisfies $\Delta_{s}$ (Goel and Ermentrout 2002):

$\Delta_{s}(\Phi)=a^{*} \sin \frac{\Pi}{1-T} * \Phi+b(1-T-\Phi)$ 


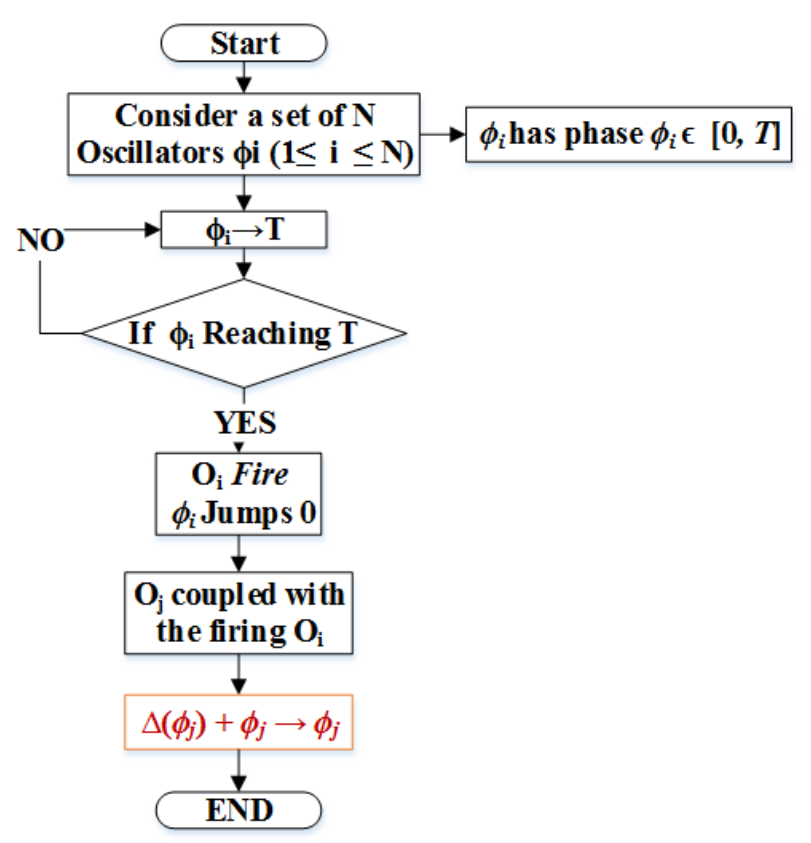

Fig. 4. Flowchart of the original PCO model

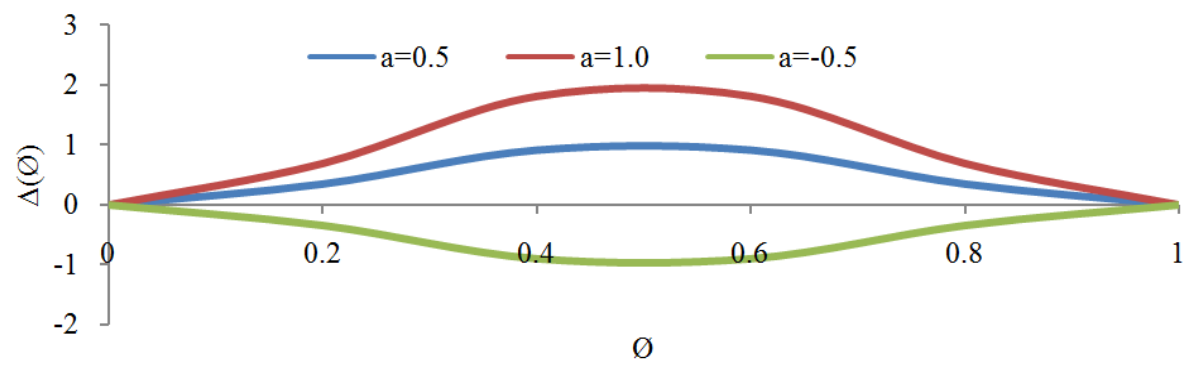

Fig. 5. QIF model

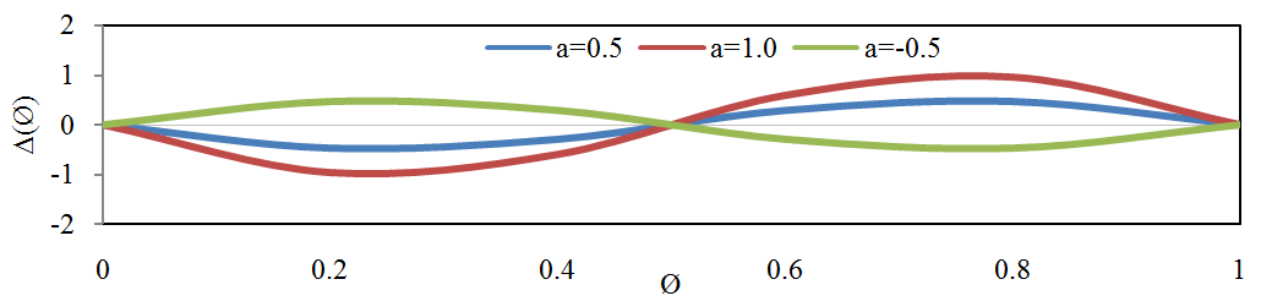

Fig. 6. RIC model

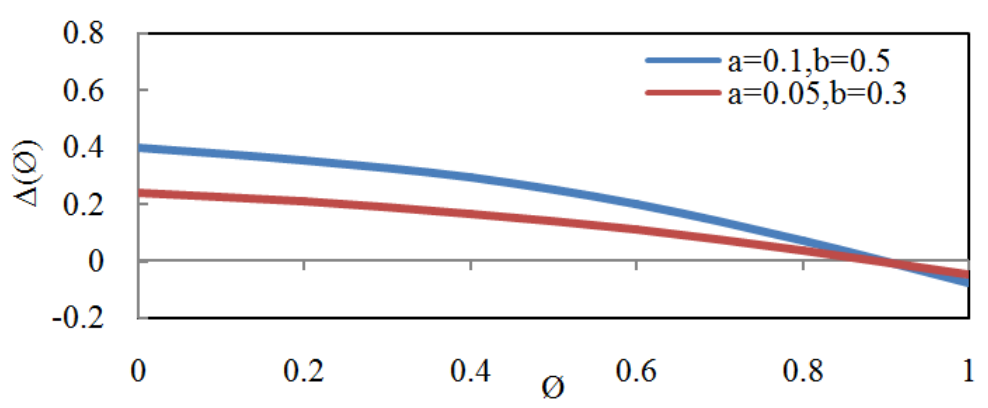

Fig. 7. PRC $\Delta_{S}(\Phi)$ 
Here, the Equation 4 based on Equation 3 with add the following:

$$
\left[\begin{array}{l}
0<\Delta\left(\Phi_{j}\right) \leq 1-T-\Phi_{j}\left(0 \leq \Phi_{j}<1-T\right) \\
\Delta\left(\Phi_{j}\right)=0\left(\Phi_{j}=1-T\right) \\
1-T-\Phi_{j} \leq \Delta\left(\Phi_{j}\right)<0\left(1-T<\Phi_{j}<1\right)
\end{array}\right.
$$

where, $a=0.1,0.05, b=0.5,0.3$ and $T=0.2$; Fig. 7 presents PRC $\Delta_{S}(\Phi)$. The PRC that satisfies RIC must lie between two lines.

\section{Wave Traveling (WT) Model}

Anchored in phase-locking in the PCO scheme, WT can systematize in data gathering and diffusion (Lanford III and Mintchev, 2015; Lv and Wang, 2010; Taniguchi et al., 2007). In this scheme, every character sensor transmits the collected data inside the phase of its own timer. Thus, whenever an SN detects transmissions from another node, the sensor network regulates the phase of its own timer consequently. Through working together with their neighbors, SNs finally enters the phase locking state in which data sensor are emitted. In such a situation, the timing of a communication that is being emitted is regarded a WT, whose center takes place at the $\mathrm{SN}$ and which seeks to either disperse/gather information to/from all SNs. Figure 8 shows the flowchart of WT in the PCO model that explains the algorithm:

$$
\Phi_{j}=\Phi_{j}+P R C_{\cdot a} * \sin \frac{\Pi}{\Gamma} * \Phi_{j}+P R C_{\cdot b}\left(\Gamma-\Phi_{j}\right)
$$

where, $P R C_{\cdot a}$ and $P R C_{\cdot b}$ were the factors to decide the rate of assemblage. Through the use of Equation 5 during the PRC function, phase locking in the PCO scheme is achieved throughout general communication among SNs regardless of their initial phase situation. Figure 9 explains the flowchart in Fig. 8 and how SNs can be classified into many categories.

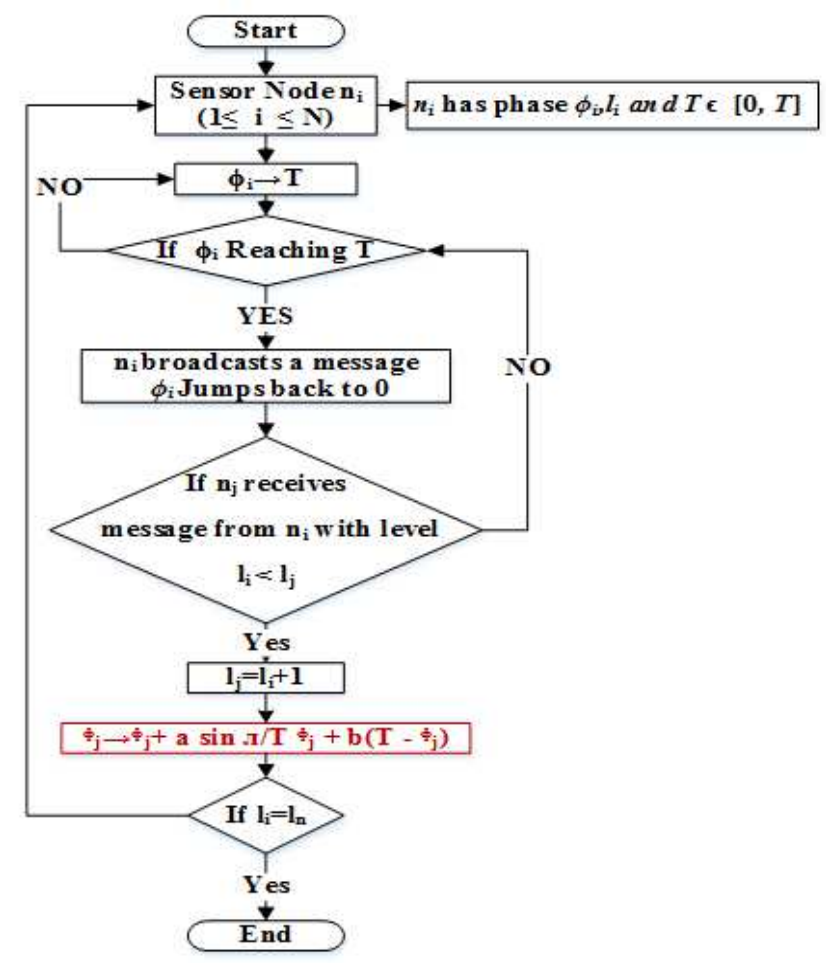

Fig. 8. Flowchart of WT in PCO

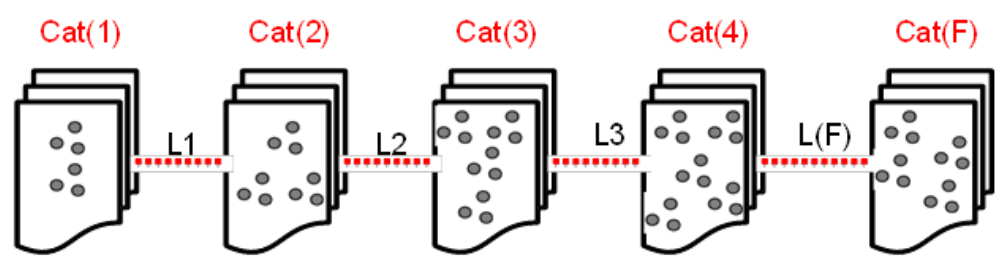

Fig. 9. Classifying the SNs into several categories 


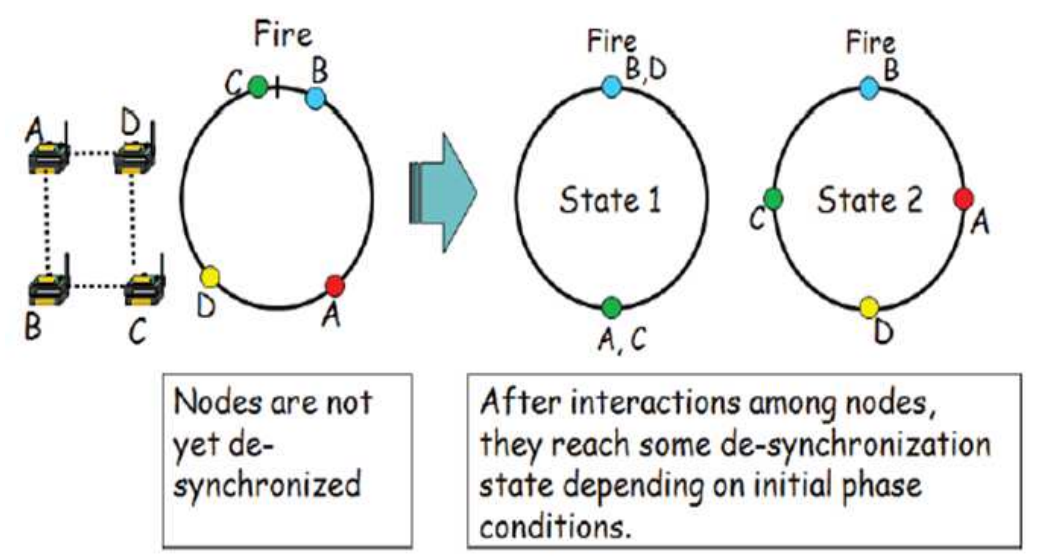

Fig. 10. DESYNC behavior of a multi-hop topology (Degesys et al., 2007)

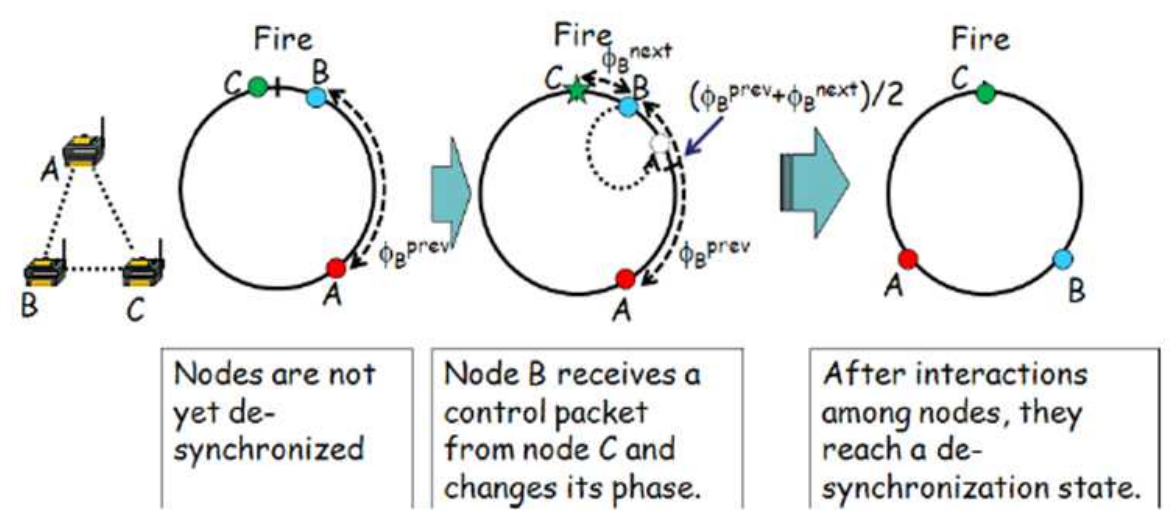

Fig. 11. DESYNC behavior of a single-hop topology (Degesys et al., 2007)

\section{DESYC Model}

DESYNC is a significant technique in the anti-phase of the planned PCO scheme (Degesys et al., 2007). DESYNC models utilize the time division multiple access technique to enable the nodes through selforganization and to allocate equally spaced timeslots regardless of topology or network size. However, the DESYNC technique cannot solve the hidden node problem, hence making this technique unsuitable for multi-hop WSNs amid increasing collision and deafness problems. This scenario is best depicted in Fig. 10, in which the first, second states of the SNs are steady depending on the original phase of individual nodes. In the former state 1, a SN and its hidden-terminals (i.e., A and $\mathrm{C}$ or $\mathrm{B}$ and $\mathrm{D}$ ) synchronize and fire concurrently, while the rest of the nodes are fired during state 2 at equally spaced intervals. SNs are unable to connect to each other during state 1. During this state, SN C couldn't obtain small packages from any SN B or SN D because of deafness and collision problems. Figure 11 presents a case of the PCO model in an anti-phase assemblage in a single-hop WSN that is collected by three SNs via DESYNC. As shown in Fig. 11, SNs arrive at a desynchronization situation after communications between SNs.

\section{The Design of Proposed Mechanism}

As shown in Fig. 12, the proposed mechanism will combine and use both phases-locking and anti-phase in PCO mechanisms, in which the phase-locking process will use WT when the SN has a data packet to propagate from the sender to the sink through the multi-hop path. In contrast, the anti-phase-based DESYNC that used TDMA protocol in the PCO model begins during the communication period to avoid deafness and collision among SNs with the same hop counts which were explained in the above section.

The Discrete Event Simulation (DES) that is divided into four events, namely, the generation of the SNs packet, dropping of the SN packet when the energy $<=0$, transmission of an SN process and receiving of the process.

\section{Generate a SN IN DES}

We generate a fundamental SN for the BS or the core-node and the neighbor $\mathrm{SNs}$ of the proposed 
methods in the sender. For estimate, the proposed scheme utilizes a WSN.

In order to explain how the BS and SN packets are generated, we generate these packets automatically by using the function Rand, use next Double () in Java Language to give the values of (REGION_X and REGION_Y) for the SNs packets and add BS as shown in Fig. 13.

After generating the BS and SNs packets, one must determine whether the radio range is in the same area or not by using the equation function of distance in Fig. 14 to check the value of parameter diff.

After verifying that all SNs packets in the same radio range are equal to 50 , the neighbor $\mathrm{SNs}$ packet is then registered as shown in Fig. 15.
After registering the SNs packet, all the parameters that are required for the $\mathrm{SN}$ packet must be initialized. The file is generated manually by using Microsoft Excel. The file has all the locations of BS for (X and $Y$ ) and the SNs region of ( $\mathrm{X}$ and $\mathrm{Y})$. The file is then read into the program buffering.

\section{Dropped the SNs}

SNs are WSNs that require every sensor data to perform events in time synchronization. This synchronization is essential for organizing power cycles, conserving power and ensuring the appropriate performance of the sensors data that calculate time sensitive events. Therefore, SNs is dropped only when their energy is equal to or less than 0 . We obtain the energy of the SNs as shown in Fig. 16.

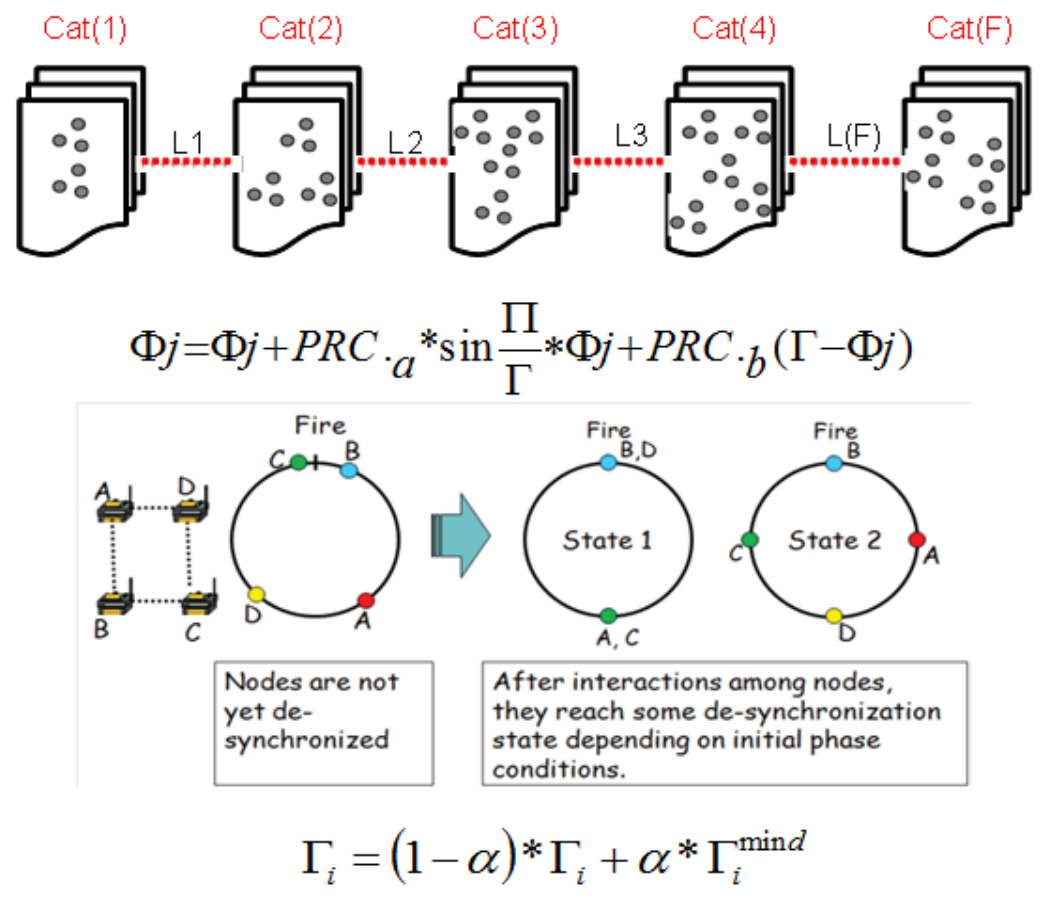

Fig. 12. Framework of the proposed scheme

$\operatorname{addBS}(0,($ double $)($ param.BS_LOCATION_X), (double) $($ param.BS_LOCATION_Y $)) ;$
$\quad$ for $(i=1 ; i<=$ node_num $;++)\{$
$x=$ rand.nextDouble ()$*($ double $)$ param.REGION_X;
$y=$ rand.nextDouble ()$*($ double $)$ param.REGION_Y;
this.addNode $(i, x, y) ;$

Fig. 13. The prototypes of function generate $\mathrm{SN}$ and $\mathrm{BS}$

private double distance (int $a$, int b) $\left\{/ / d=\operatorname{sqrt}\left((x 2-x 1)^{\wedge} 2+(y 2-Y 1)^{\wedge} 2\right)\right.$

return Math.sqrt(Math.pow(node[a].x() - node[b].x(0,2) + Math.pow(node[a].y( - node[b].y(0,2));\}

Fig. 14. The prototypes of equation function for distance 


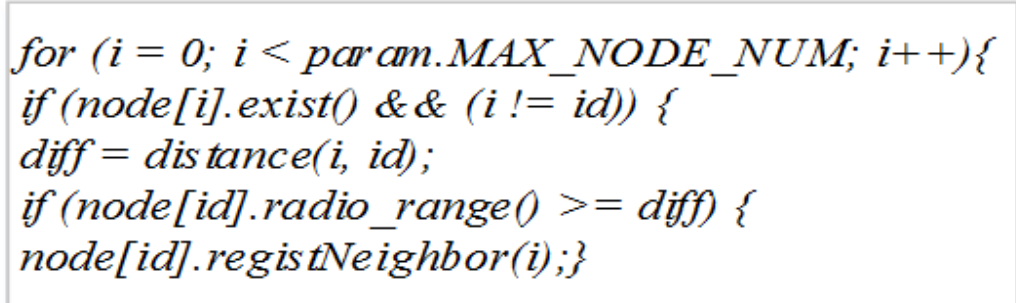

Fig. 15. The prototypes of the function for registering the neighbor SNs

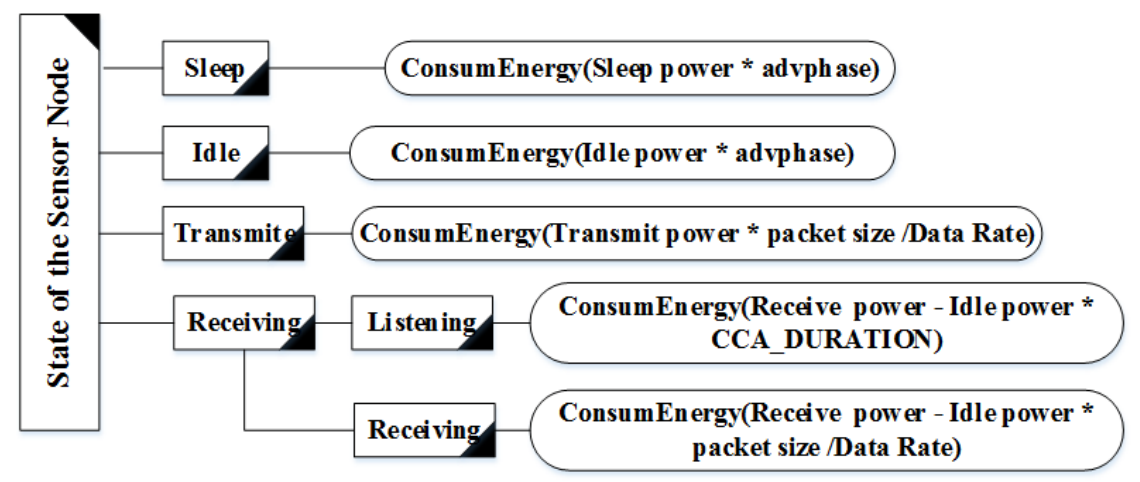

Fig. 16 Obtaining the energy of the SNs

\section{Transmitting a Process}

SN behavior: The behavior of the sent message following a number of cycles and following the sensor nodes reaches the stable state. As mentioned earlier, the behavior of the $\mathrm{SN} n_{i}$ is in agreement with its current phase $\Phi_{i}$ and the SN receives manage messages from its neighboring nodes.

Wake-up: The SN $n_{i}$ enters the wakeup state when the phase $\Phi_{i}=\Gamma-\tau^{\max }$. During this phase, the node empties the communication broadcast timing table and sensor data $D_{i}$ and then waits for messages to be received from its neighbors.

Message reception from downstream nodes: After receiving a message from its downstream node $n_{j}$ at time $\tau$ where $l_{j}=l_{i}+1$, th.ode adds the newly arrived data $D_{j}$ to its to its overall data $D_{i}$. Similarly, the node $n j$ denotes a new entry $e_{i, j}=\left(j, l_{j}, \tau\right)$ into its data transmission timing table $\varepsilon_{i}$.

Message reception from same hop nodes: After receiving a message from another node at time $\tau$ where $l_{j}$ $=l_{i}$, the .e adds a new entry $e_{i, j}=\left(j, l_{j}, \tau\right)$ into its data transmission timing table $\varepsilon_{i}$.

Message transmission: After the phase $\Phi_{i}=\Gamma$, the node $n_{i}$ transmits a broadcast packet to all other nodes in the wake-up state and within the transmission range. When $\Gamma$ is reached, the phase $\Phi i$ is reset to zero and time $\tau$ is recorded as the transmission time $t_{i}^{\text {trans }}$.
When transmitting the message, the CSMA/CA technique is employed.

Message reception from upstream nodes: Upon resetting to zero, the node $n_{i}$ remains in the wakeup state for $\Gamma^{\max }$. Upon receiving a message from its upstream node $n_{j}$ at time $\tau$ where $l_{j}<l_{i}$, the node $n_{i}$ adjusts its level as $l_{i}=l_{j}+1$ and its phase is shifted in accordance with Equation 5.

\section{Receiving the Process}

Upon receiving a message and when the condition $l_{j}$ $=l_{i}+1$ is satisfied, the transmission timing table $\varepsilon_{i}$ of node $n_{i}$ is updated as follows. First, the transmission timing information of the received message $F_{j}$ is utilized to estimate the message trthe nodession time $t_{j, k}^{\text {trans }}=\tau-f_{j, k}$ of node $n_{k}$, where $n_{k}$ denotes a one- or twohop neighbor of node $n_{i}$. When the node $n_{k}$ has no entries in its message tran,sthe node timing table $\varepsilon_{i}$, node $n_{i}$ creates a new entry $e_{i, k}=\left(k, l_{i}, \tau_{j, k}^{\text {trans }}\right)$ in the table. By contrast, when a node $n_{k}$ has an entry in its message transmission timing table $\varepsilon_{i}$ and when the condition $t_{i, k}^{\text {trans }}>t_{j, k}^{\text {trans }}$ is satisfied, node $n_{i}$ overwrites the entry as $e_{i, k}=\left(k, l_{i}, \tau_{j, k}^{\text {trans }}\right)$.

When phase reaches $\tau^{\max }$ and node $n_{i}$ is stimulated, the node updates its offset $\Gamma_{i}$. Otherwise, the node has to wait for stimulation, which is attained after receiving a 
message from an upstream node. The node $n_{i}$ is associated with a $\tau_{i}^{\text {prev }}$ and $\tau_{i}^{\text {next }}$ as follows:

$$
\begin{gathered}
\tau_{i}^{\text {prev }}==_{\tau \in \Gamma_{i, t}^{\max }<\tau_{i}^{\text {trans }}} \tau \\
\tau_{i}^{\text {prev }}={ }_{\tau \in \Gamma_{i, t}^{\min }<\tau_{i}^{\text {trans }}} \tau
\end{gathered}
$$

where, $\Gamma_{i}$ denotes the set of time estimates transmission messages in $\varepsilon_{i}$. In the where $\tau_{i}^{\text {prev }}$ or $\tau_{i}^{\text {next }}$ are not attained, they are assumed to be zero. The offset $\tau_{i}$ is then adjusted by node $n_{i}$ based on the relation:

$$
\Gamma_{i}=(1-\alpha)^{*} \Gamma_{i}+\alpha^{*} \mathrm{~T}_{i}^{\text {mid }}
$$

Where:

$$
\begin{aligned}
& \tau_{i}^{\min d}=\left\{\begin{array}{l}
\frac{\Gamma_{i}^{\text {prev }}+\Gamma_{i}^{\text {next }}}{2} \text { if } \tau_{i}^{\text {next }}>\tau_{i}^{\text {prev }}>0 \\
\frac{\Gamma_{i}^{\text {prev }}}{2} \text { if } \tau_{i}^{\text {next }}=0, \tau_{i}^{\text {prev }}>0 \\
\Gamma^{\max } \text { otherwise, }
\end{array}\right. \\
& \Gamma_{i}^{\text {prev }}=\tau_{i}^{\text {stim }}-\tau_{i}^{\text {prev }} \\
& \Gamma_{i}^{\text {next }}=\tau_{i}^{\text {stim }}-\tau_{i}^{\text {next }}
\end{aligned}
$$

\section{Performance Evaluation}

Based on the highlighted procedure, several simulation runs were executed to prove the superior recital of the planned scheme under various WSN conditions as well as to minimize the deafness and collision problems that are associated with WSNs.

\section{Simulation Environment}

The suggested environment concerned a fixed $100 \times 100 \mathrm{~m}$ square field where sensor nodes were arbitrarily deployed. The BS was situated at the $50 \times 50 \mathrm{~m}$ mark. The broadcast radius of the sensor nodes was 50 $\mathrm{m}$, which was unspecified to be completely omnidirectional. Thus, at any time a node sent broadcast communication from two dissimilar nodes at the same time, the receiver node would be ineffective to receive both messages because of a collision or deafness. The data assembly ratio cycle, $T$, was set to $1 \mathrm{~s}$, while the uppermost alternate, $\tau^{\max }$, was set to $0.1 \mathrm{~s}$. From Equation $5, a, b$ and $\alpha$ were set to $0.01,0.5$ and 0.5 , correspondingly. The preliminary phase interpretations were randomly set. The sizes of the communication header and that of the individual sensor data were set to $2 \mathrm{~B}$, whereas that of the communication broadcast control information was set to $1 \mathrm{~B}$. The CSMA/CA factors included a back-off time slot of $1 \mathrm{~ms}$ with a back- off of $4 \mathrm{~ms}$. The simulation was performed in a high-end Java simulator for wireless communication. A summary of the other parameters that are utilized in the simulation is displayed in Table 1.

\section{Performance Metrics Evaluation}

The performance metrics that are discussed in this subsection include energy efficiency ratio and data gathering ratio.

\section{Energy Efficiency Ratio}

The ratio of the total consumed energy to the number of packets that are received by the sink node. This ratio is calculated as follows:

$$
\text { Energy efficiency }=\frac{\sum_{i=1}^{n} \operatorname{ToEnc}(i)}{\text { Topck }_{\text {Sink }}}
$$

where, $n$ represents the number of SNs in the network, ToEnc( $i)$ is the total energy consumption for each SN $i$ and $T_{o p c k_{\text {Sink }}}$ is the total number of packets that are received by the sink node.

\section{Data Gathering Ratio}

The ratio of the total of $\mathrm{SN}$ data that are gathered at the BS per cycle. This ratio is calculated as follows:

$$
\frac{\sum_{i=1}^{n} T_{o p c k_{S i n k}}}{S N C}
$$

where, $n$ represents the number of SNs in the network, Topck $_{\text {Sink }}$ is the total number of packets that are received by the sink node for each SN I and SNC is the SNs per cycle.

\section{Discussion of the Experimental Results}

The results that are obtained using the proposed PCO technique for minimizing collision and deafness problems are then compared with those that are obtained using the WT based technique. The results were classified into two groups according to the effect of the node packet and that of the packet size in the superior performance of the proposed technique.

\section{The Effect of the Number of SNs}

Figure 17 shows the data gathering ratio of both the proposed scheme and the WT scheme. The data gathering ratio of the proposed scheme decreases when the digit of deployed nodes exceeds 30 , but rises to $100 \%$ when the number of deployed nodes is below 30 . This development of the proposed scheme is credited to the fact that the proposed method considers the hidden node problem. 
Zeyad Ghaleb Al-Mekhlafi et al. / Journal of Computer Sciences 2016, 12 (10): 482.494 DOI: $10.3844 /$ jcssp.2016.482.494

Table 1. Parameters setup

\begin{tabular}{|c|c|c|}
\hline \multirow[b]{2}{*}{ Parameter } & \multicolumn{2}{|l|}{ Value } \\
\hline & Scenario I & Scenario II \\
\hline Channel Frequency & $2.4 \mathrm{GHz}$ & $2.4 \mathrm{GHz}$ \\
\hline Number of nodes & $10.20,30,40,50,60,70,80,90,100$ & 30 \\
\hline MIN_TIME_STEP & 0.00001 & 0.00001 \\
\hline Packet Data Size & 16 bits & $8,16,40,80,160,400,800$ bits \\
\hline Energy Model & $\mathrm{MICAz}$ & $\mathrm{MICAz}$ \\
\hline Data rate & $250 \mathrm{kbps}$ & $250 \mathrm{kbps}$ \\
\hline Transmit Power & $52.2 \mu \mathrm{W}$ & $52.2 \mu \mathrm{W}$ \\
\hline Receive Power & $59.1 \mu \mathrm{W}$ & $59.1 \mathrm{~mW}$ \\
\hline Idle Power & $60 \mu \mathrm{W}$ & $60 \mu \mathrm{W}$ \\
\hline Sleep power & $3 \mu \mathrm{W}$ & $3 \mu \mathrm{W}$ \\
\hline INITIAL_ENERGY & 100 Joules & 100 Joules \\
\hline
\end{tabular}

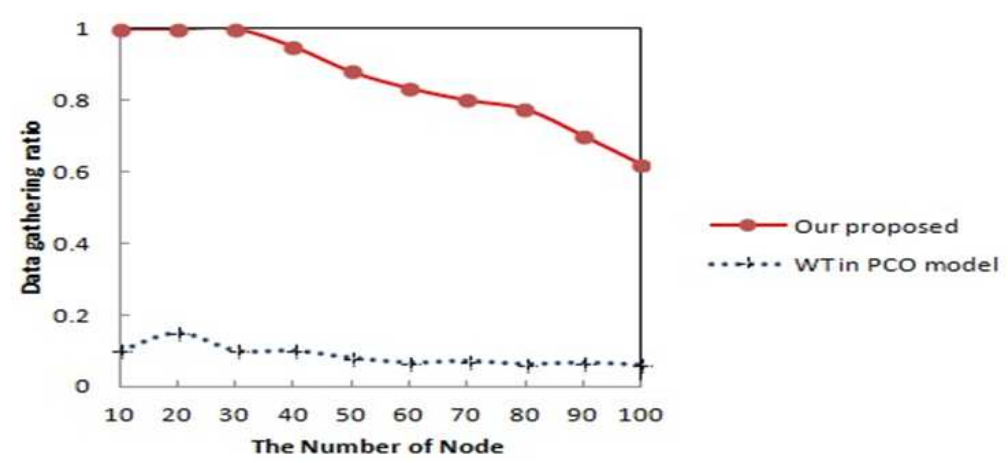

Fig. 17. Comparison of the data gathering ratio at the sink between the proposed scheme and WT

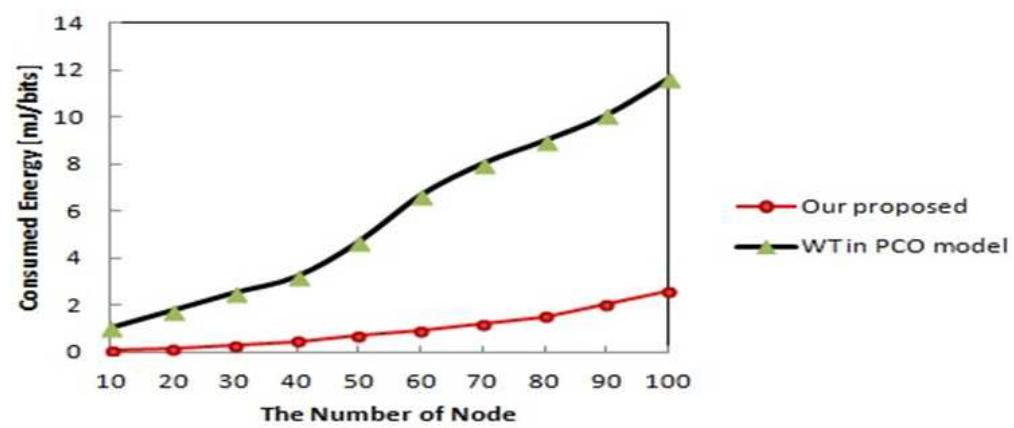

Fig. 18. Comparison of the energy consumption ratio between the proposed scheme and WT

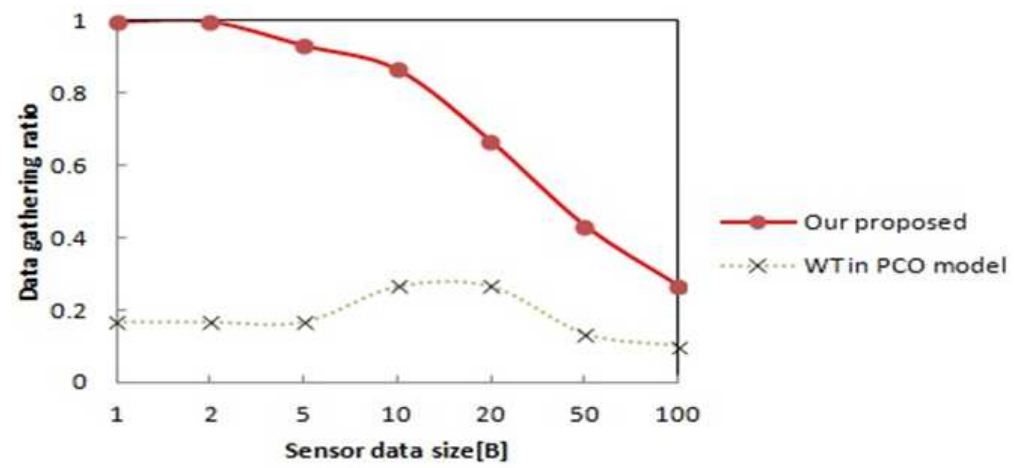

Fig. 19. The data gathering ratio of the proposed model and the WT at $30 \mathrm{SNs}$ based on data size 


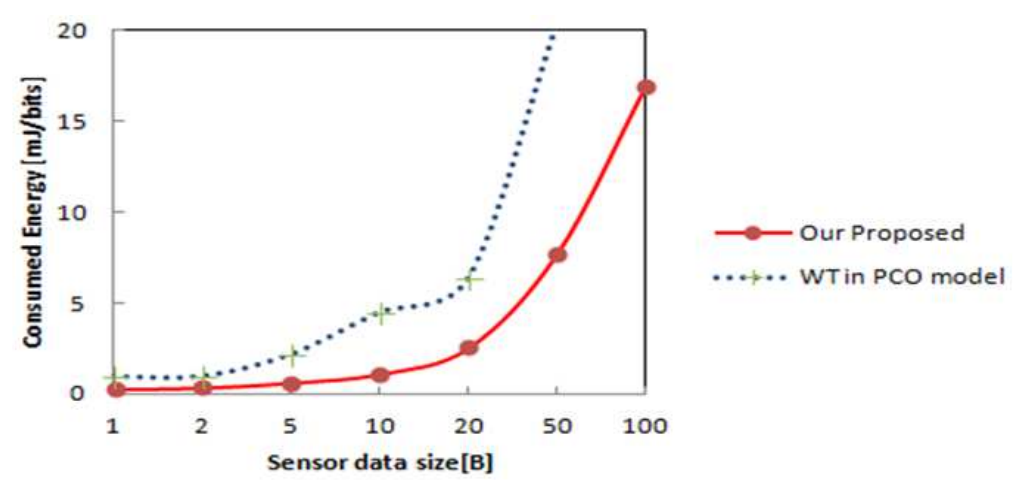

Fig. 20. Consumed energy of the proposed scheme and WT at 30 SNs based on data size

Figure 18 shows that the proposed mechanism performs much better than the WT in terms of energy efficiency because of the higher number of received packets in the former. Accordingly, the consumed power of the WT in PCO method is superior then that of the proposed scheme.

Figure 19 and 20 demonstrate the data assembly and consumed power per SN datum size, correspondingly, of both models when 30 nodes are deployed. Despite of SN data size, the proposed scheme is superior over the WT scheme. Therefore, the proposed scheme is appropriate for WSN applications with advanced data assembly ratio. Nevertheless, the scheme involves extra control overheads that are required for avoiding collision and deafness problems.

\section{Conclusion}

Guaranteeing connectivity and monitoring the observed area are highly demanded in WSN applications. This paper presented a self-organizing time synchronization algorithm that was adopted from the traditional PCO model of fireflies flashing synchronization in order to counteract deafness and collision in terms of data assembly ratios and energy efficiency. The simulation outcome demonstrate that the suggested scheme outperforms the WT scheme, which confirms the impact of the proposed mechanism for WSNs. Future studies must corroborate and get better the recital of these schemes by using the reachback of firefly algorithm in actual environments and experimental evaluations.

\section{Acknowledgment}

We would like to thank Dr. Mohammad Al-Shameri and Dr. Ahmed M. Shamsan Saleh for providing constructive suggestions and feedback that helped in improving the quality of mathematics in the paper.

\section{Funding Information}

This work was supported by Fundamental Research Grant Scheme (FRGS) UPM-FRGS-08-02-13-1364FR.

\section{Author's Contributions}

Zeyad Ghaleb Al-Mekhlafi: Participated in all experiments, designed the research plan, organized the study, methodology and contributed to the writing of the manuscript.

Zurina Mohd Hanapi: Coordinated the mouse work, designed the research plan, organized the study, methodology and evaluation.

Mohamed Othman: Coordinated the mouse work and evolution the whole paper.

Zuriati Ahmad Zukarnain: Coordinated the mouse work and evolution the whole paper.

\section{Ethics}

This article is original and contains unpublished materials. The corresponding author confirms that all of the other authors have read and approved the manuscript and no ethical issues involved.

\section{References}

Al-Mekhlafi, Z.G., Z.M. Hanapi, M. Othman, Z.A. Zukarnain and A.M.S. Saleh, 2014a. Energy efficient on aspect of clock synchronization in a wireless sensor network. J. Applied Sci., 14: 1101-13. DOI: 10.3923/jas.2014.1101.1113

Al-Mekhlafi, Z.G., Z.M. Hanapi, M. Othman, Z.A. Zukarnain and F. Hashim et al., 2014b. Impact of the deafness problem on clock synchronization in a wireless sensor network. Proceedings of the 6th International Conference on Management of Emergent Digital EcoSystems, Sept. 15-17, ACM, Saudi Arabia, pp: 127-32. DOI: $10.1145 / 2668260.2668261$

Brunel, N. and P.E. Latham, 2003. Firing rate of the noisy quadratic integrate-and-fire neuron. Neural Comput., 15: 2281-306. DOI: $10.1162 / 089976603322362365$

Buck, J., 1988. Synchronous rhythmic flashing of fireflies. II. Q. Rev. Biol., 63: 265-89. DOI: 10.1086/415929 
Buck, J., E. Buck, J.F. Case and F.E. Hanson, 1981. Control of flashing in fireflies. J. Comparative Physiol., 144: 287-98. DOI: 10.1007/BF00612560

Canavier, C.C. and S. Achuthan, 2010. Pulse coupled oscillators and the phase resetting curve. Math. Biosci., 226: 77-96. DOI: 10.1016/j.mbs.2010.05.001

Degesys, J., I. Rose, A. Patel and R. Nagpal, 2007. DESYNC: Self-organizing desynchronization and TDMA on wireless sensor networks. Proceedings of the 6th International Conference on Information Processing in Sensor Networks, Apr. 25-27, IEEE Xplore Press, pp: 11-20. DOI: 10.1109/IPSN.2007.4379660

Goel, P. and B. Ermentrout, 2002. Synchrony, stability and firing patterns in pulse-coupled oscillators. Phys. D: Nonlinear Phenomena, 163: 191-216. DOI: $10.1016 / \mathrm{S} 0167-2789(01) 00374-8$

Hong, Y.W. and A. Scaglione, 2005. A scalable synchronization protocol for large scale sensor networks and its applications. IEEE J. Selected Areas Commun., 23: 1085-99. DOI: 10.1109/JSAC.2005.845418

Lanford III, O.E. and S.M. Mintchev, 2015. Stability of a family of travelling wave solutions in a feedforward chain of phase oscillators. Nonlinearity, 28: 237. DOI: $10.1088 / 0951-7715 / 28 / 1 / 237$

Leidenfrost, R. and W. Elmenreich, 2008. Establishing wireless time-triggered communication using a firefly clock synchronization approach. Proceedings of the International Workshop on Intelligent Solutions in Embedded Systems, Jul. 10-11, IEEE Xplore Press, pp: 1-18.

DOI: 10.1109/WISES.2008.4623299

Lucarelli, D. and I.J. Wang, 2004. Decentralized synchronization protocols with nearest neighbor communication. Proceedings of the 2nd International Conference on Embedded Networked Sensor Systems, Nov. 03-05, ACM, Baltimore, MD, USA., pp: 62-68. DOI: 10.1145/1031495.1031503

Lv, G. and M. Wang, 2010. Traveling waves of some integral-differential equations arising from neuronal networks with oscillatory kernels. J. Math. Anal. Applic., 370: 82-100. DOI: $10.1016 /$ j.jmaa.2010.04.011

Mathar, R. and J. Mattfeldt, 1996. Pulse-coupled decentral synchronization. SIAM J. Applied Math., 56: 1094-106. DOI: 10.1137/S0036139994278135
Mirollo, R.E. and S.H. Strogatz, 1990. Synchronization of pulse-coupled biological oscillators. SIAM J. Applied Math., 50: 1645-62. DOI: 10.1137/0150098

Nishimura, J., 2015. Frequency adjustment and synchrony in networks of delayed pulse-coupled oscillators. Phys. Rev. E, 91: 012916-012916. DOI: 10.1103/PhysRevE.91.012916

Nokhanji, N., Z.M. Hanapi, S. Subramaniam and M.A. Mohamed, 2015. An Energy Aware Distributed Clustering Algorithm Using Fuzzy Logic for Wireless Sensor Networks with Non-uniform Node Distribution. Wireless Personal Commun., 84: 395-395. DOI: 10.1007/s11277-015-2614-9

Nunez, F., Y. Wang and F.J. Doyle, 2012. Bio-inspired hybrid control of pulse-coupled oscillators and application to synchronization of a wireless network. Proceedings of the American Control Conference, Jun. 27-29, IEEE Xplore Press, pp: 2818-23. DOI: 10.1109/acc.2012.6314981

Taniguchi, Y., N. Wakamiya and M. Murata, 2007. A traveling wave based communication mechanism for wireless sensor networks. J. Netw., 2: 24-32. DOI: $10.4304 /$ jnw.2.5.24-32

Tyrrell, A., G. Auer and C. Bettstetter, 2006. Fireflies as role models for synchronization in ad hoc networks. Proceedings of the 1st International Conference on Bio Inspired Models of Network, Information and Computing Systems, Dec. 11-13, ACM, Cavalese, Italy. DOI: $10.1145 / 1315843.1315848$

Tyrrell, A., G. Auer and C. Bettstetter, 2007. Biologically Inspired Synchronization for Wireless Networks. In: Advances in Biologically Inspired Information Systems, Dressler, F. and I. Carreras (Eds.), Springer Berlin Heidelberg, pp: 47-62.

Wang, Y., F. Nunez and F.J. Doyle, 2012. Energyefficient pulse-coupled synchronization strategy design for wireless sensor networks through reduced idle listening. IEEE Trans. Signal Process., 60: 5293-306. DOI: 10.1109/TSP.2012.2205685

Werner-Allen, G., G. Tewari, A. Patel, M. Welsh and R. Nagpal, 2005. Firefly-inspired sensor network synchronicity with realistic radio effects. Proceedings of the 3rd International Conference on Embedded Networked Sensor Systems, Nov. 02-04, ACM, San Diego, California, USA., pp: 142-53. DOI: $10.1145 / 1098918.1098934$ 